\title{
Using basic technology - and corporate social responsibility - to save lives
}

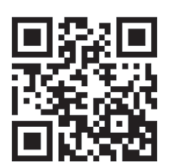

While South Africa (SA) will almost certainly fail to meet next year's Millennium Development Goals (MDGs) of reducing deaths of children under 5 by two-thirds and the maternal mortality rate by three-quarters, one brilliantly simple technological innovation is accelerating progress.

It's called 'MomConnect' - a free SMS (text message) pregnancy information and advice service targeting the 1.2 million women who fall pregnant every year (one million using the public sector and 200000 the private). After registering at their clinic or private health facility, the expectant (and existing) mothers get SMSed fortnightly advice and information tailored to their stage of pregnancy, or appropriate to their newborn infant or child of up to 18 months. For hundreds of thousands of pregnant women, many in far-flung, poverty-stricken rural areas or in their vulnerable teenage years, the service is a veritable lifeline. Teenage girls, for example, many of them first-time mothers, account for a disproportionate $36 \%$ of maternal deaths, while representing only $8 \%$ of all annual pregnancies.

The National Department of Health $(\mathrm{NDoH})$, realising that more South Africans use a mobile phone than watch television or listen to the radio (there are more SIM cards than people), decided it was time to knock on the doors of the locally operating cell phone companies to see whether they would help make the initiative fly. Vodacom, MTN, Cell C and Telkom all promptly agreed

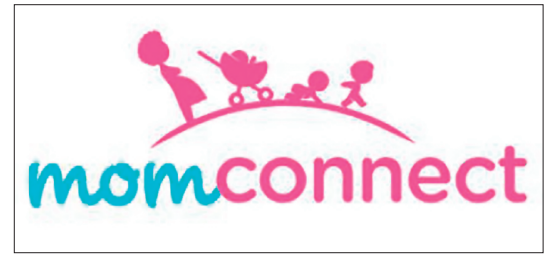

to provide a $50 \%$ discount on all SMSs sent to MomConnect-registered mothers. The NDoH also secured R49 million in funding from the US government and R5 million each from Johnson \& Johnson and ELMA Philanthropies, thus retaining its own precious budget for other vital lifesaving interventions that have finally and belatedly begun to make an impact on the shocking death statistics for mothers and children. National health minister 
Dr Aaron Motsoaledi conducted a national MomConnect tour this August, addressing many of the $10 \quad 000$ public healthcare workers newly trained in the programme and checking that every health facility had a dedicated registration facilitator, backed by a provincial counterpart and co-ordinated nationally.

\section{Presenting in labour is too often fatally late}

'Many women end up with a complicated pregnancy simply because they didn't know what to do,' explained Motsoaledi. 'You [the healthcare workers] are helpless to change the outcome if their first point of contact is the labour ward,' he emphasised. 'This way we can give advice and share what could be lifesaving knowledge during pregnancy - and afterwards - on exclusive breastfeeding, nutrition, immunisation, oral rehydration during diarrhoea - not to mention family planning.' Complications can be identified early, and information on safe abortions and future contraception come with the package. The service also crucially reminds its users when they are due for a clinic check-up. Between its launch in October (Women's Month) this year and 23 October, some 103000 women had enrolled, with the number increasing at a rate of more than 10000 per week and $69 \%$ of clinics participating, according to the NDoH. Currently available in English, Afrikaans, Zulu, Xhosa, Sotho and Tswana, the service will be available in all 11 official languages by early next year, an $\mathrm{NDoH}$ spokesman added.

\section{Complications can be identified early, and information on safe abortions and future contraception come with the package. The service also crucially reminds its users when they are due for a clinic check-up.}

Maternal deaths in SA climbed steadily from 1998, when 150 mothers died per 100000 live births, to 2009, when the figure reached 312 , before dropping back to
269 in late 2010 - after the 2009 findings of the health ministry's Confidential Enquiry into Maternal Deaths began to be speedily acted upon. It now stands at 140, making SA's MDG target of 38 for the end of next year 'a possible or impossible dream', as Motsoaledi put it, in a seemingly clumsy attempt to sound both upbeat and realistic. The Saving Babies 2010 - 2011 report showed the early neonatal death rate to be $21 / 1000$ live births, with the majority of these deaths occurring in the 1000 $1499 \mathrm{~g}$ weight category. SA has reduced the overall under-5 child mortality rate from 61/1 000 births in 1990 (the MDG inception date) to 45 currently. Reaching the MDG goal of 20 would need more to be done in the next 16 months than was done in the past 24 years.

\section{Bright light at the end of the tunnel}

Yet SA has much cause for hope. The largest HIV testing and antiretroviral (ARV) rollout campaign the world has yet seen, plus a massive push on the programme for prevention of mother-to-child transmission (PMTCT) of HIV over the past 10 years, now has over $98 \%$ of public health facilities providing these vital services. An estimated 99\% of women receive an HIV test during pregnancy, and $93 \%$ of HIV-positive mothers are receiving ARV treatment or prophylaxis. A national evaluation involving 10178 infants in 572 health facilities showed that the 6-week vertical HIV transmission rate now stands at $2.7 \%$, compared with rates of $20-30 \%$ less than 10 years ago.

So just how will an intervention like MomConnect impact? Only proper research will eventually tell, but for now it will encourage pregnant women to start antenatal care early and enable testing for the killers of hypertension, HIV/ AIDS and diabetes very early on in their pregnancy, thus significantly boosting an already successful PMTCT campaign. The MomConnect programme has an additional benefit; mothers will be able to send the $\mathrm{NDoH}$ free SMS messages (a 'please call me') to share their concerns about and experiences of public healthcare facilities, providing an invaluable 'feedback loop' for potential improvement as the national campaign to improve public sector caregiving standards gathers momentum. It's also the answer to tracking SA's highly mobile population, enabling healthcare workers to plug into their patients' history by linking the existing medical registry to an electronic database that can be accessed from all MomConnect clinics. This will mean that a woman can be more effectively treated at any participating facility.

\section{After registering at their clinic or private health facility, the expectant (and existing) mothers get SMSed fortnightly advice and information tailored to their stage of pregnancy, or appropriate to their newborn infant or child of up to 18 months.}

One independent mobile strategy firm, Tomi Ahonen Consulting, gave an insight into how smartphones (comprising 21\% of SA's mobile phones, but not required for MomConnect) can additionally broaden the educational reach of users. By exploiting the Android youth platform and free instant messaging service Mxit Reach, educational packages such as Babyinfo provide free basic day-to-day updates about pregnancy and tips on how to make a pregnancy more comfortable. Paying only for the internet connection, users can also download counselling applications such as Angel and LoveLife (substance abuse and HIV counselling, respectively). Mxit Reach is only one of several applications. Others include Hi4Life ('information on HIV, pregnancy and baby health', an initiative co-ordinated by the organisation HIVSA with financial support from the Elton John Foundation). There are over 100 mobile health services of varying sizes, focuses and quality currently on offer in SA. It's a classic example of how user-friendly First-World technology can help uplift and support Third-World communities - and save countless thousands of lives.

\section{Chris Bateman}

chrisb@hmpg.co.za

S Afr Med J 2014;104(12):839-840. DOI:10.7196/SAMJ.9118 